J3eA, Journal sur l'enseignement des sciences et technologies de l'information et des systèmes, Volume 4, Hors-Série 2, 20 (2005)

DOI : http://dx.doi.org/10.1051/bib-j3ea:2005720

(C) EDP Sciences, 2005

Projet de synthèse de l'électronique analogique : réalisation d'une balance à jauges de contrainte

P. Lagonotte, J.P. Le Bourg, T. Doget et J.P. Allard

IUT de Poitiers

Département G.E.I.I.

6 allée Jean Monnet

F-86010 Poitiers CEDEX, France 


\title{
PROJET DE SYNTHÈSE DE L'ÉLECTRONIQUE ANALOGIQUE : RÉALISATION D'UNE BALANCE À JAUGES DE CONTRAINTE
}

\author{
P. Lagonotte, J.-P. Le Bourg, T. Doget, J.-P. Allard, \\ I.U.T. de Poitiers, Département G.E.I.I., 6, allée Jean Monnet, 86010 Poitiers cedex
}

\begin{abstract}
Résumé :
Nous présentons dans ce document un projet d'électronique qui a la particularité d'être à la fois concret et appliqué à la réalisation d'un « instrument de mesures » (une balance à jauges de contrainte), et d'autre part d'être suffisamment simple pour des élèves de $1^{\text {ère }}$ année et suffisamment ambitieux pour mettre en œuvre différents montages à amplificateurs opérationnels étudiés lors du cours et des travaux dirigés.

L'étude détaillée du montage électronique est présentée dans ce document ainsi que le schéma global du montage. Une part de ce projet est consacrée à la prise en main d'un logiciel de C.A.O. par les étudiants allant de la saisie de schéma jusqu'au routage du circuit imprimé. Ce projet est également l'occasion d'aborder des problèmes technologiques comme les défauts et les tolérances des différents composants, les réglages par potentiomètres, le découplage des alimentations, l'implantation et l'accessibilité des différents éléments.
\end{abstract}

Mots clés : électronique analogique, instrumentation, jauges de contrainte, projet $1^{\text {ère }}$ année d'I.U.T.

\section{INTRODUCTION}

Le choix d'un projet d'électronique au premier semestre de première année d'I.U.T GEII n'est pas toujours simple. Il est nécessaire de trouver le meilleur compromis entre :

- un sujet suffisamment simple pour pouvoir être réalisé avec un nombre limité de séances (36h);

- un sujet suffisamment ambitieux pour pouvoir illustrer un nombre important de fonctions de l'électronique analogique et permettre une étude théorique intéressante.

Ce sujet doit :

- permettre une initiation à la saisie d'un schéma électronique, à sa simulation et au routage ;

- permettre une réalisation simple suivie d'essais, de réglages et de mesures ;

- avoir un prix de revient compatible avec un budget à respecter.

Le domaine de l'instrumentation et des capteurs offre une variété de systèmes intéressants à exploiter. De plus, ce domaine permet l'étude de phénomènes physiques et leur mise en forme pour devenir exploitable au niveau d'une acquisition de données $( \pm 10 \mathrm{~V})$. Nous tenons à souligner que bien que le numérique prenne dans nos enseignements une place de plus en plus prépondérante, l'électronique analogique conserve une relation très forte et nécessaire avec la physique.

\section{«Sans analogique plus de physique! ».}

Nous avons choisi de mettre en œuvre des jauges de contrainte facilement disponibles dans le commerce et très utilisées dans la mesure des contraintes mécaniques.

\section{LE SYSTÈME RÉALISÉ}

Pour rendre cette étude plus intéressante et concrète, nous avons choisi de prendre le classique problème de la poutre encastrée en R.D.M. (résistance des matériaux) et de le transformer en une balance de mesure de masse. La poutre est réalisée en tôle de dural de $3 \mathrm{~mm}$ d'épaisseur, 20 $\mathrm{mm}$ de largeur et de $250 \mathrm{~mm}$ de longueur (figure 1).

Nous utilisons des jauges standardisées à $\mathrm{Rp}=120$ $\Omega$ collées au plus près de l'encastrement de manière à avoir le maximum de sensibilité. Un pont à une seule jauge est sensible à la température et manque de sensibilité (figure 2). Un pont à deux jauges est deux fois plus sensible que le précédent (1 jauge en extension, 1 jauge en compression) et devient insensible à la température. Un pont à quatre jauges est quatre fois plus sensible que le premier (2 jauges en extension, 2 jauges en compression) et insensible à la température. Cependant, considérant la difficulté de collage des jauges et par souci d'économie, nous avons choisi d'utiliser des ponts de Wheatstone à deux jauges. Compte tenu de la dissipation permise dans les jauges, la tension d'alimentation du pont est fixée à $\mathrm{Vp}=2,4 \mathrm{~V}$.

Dans ces conditions, la différence de potentiel (Va - $\mathrm{Vb}$ ) délivrée par le pont se décompose en deux composantes :

- celle due à la contrainte: $\mathrm{Vp}(\delta \mathrm{Rp} / 2 \mathrm{Rp})$; environ $1,2 \mathrm{mV}$ avec $\delta \mathrm{Rp}$ de l'ordre de $0,1 \%$;

- celle due à l'erreur naturelle sur les résistances du pont: $\mathrm{Vp}(\Delta \mathrm{Rp} / \mathrm{Rp}) ; 12 \mathrm{mV}$ avec $\Delta \mathrm{Rp}$ de $0,5 \%$.

Cette erreur sera donc à compenser ainsi que celles dues aux tensions de décalage e des amplificateurs comme nous le verrons par la suite. 


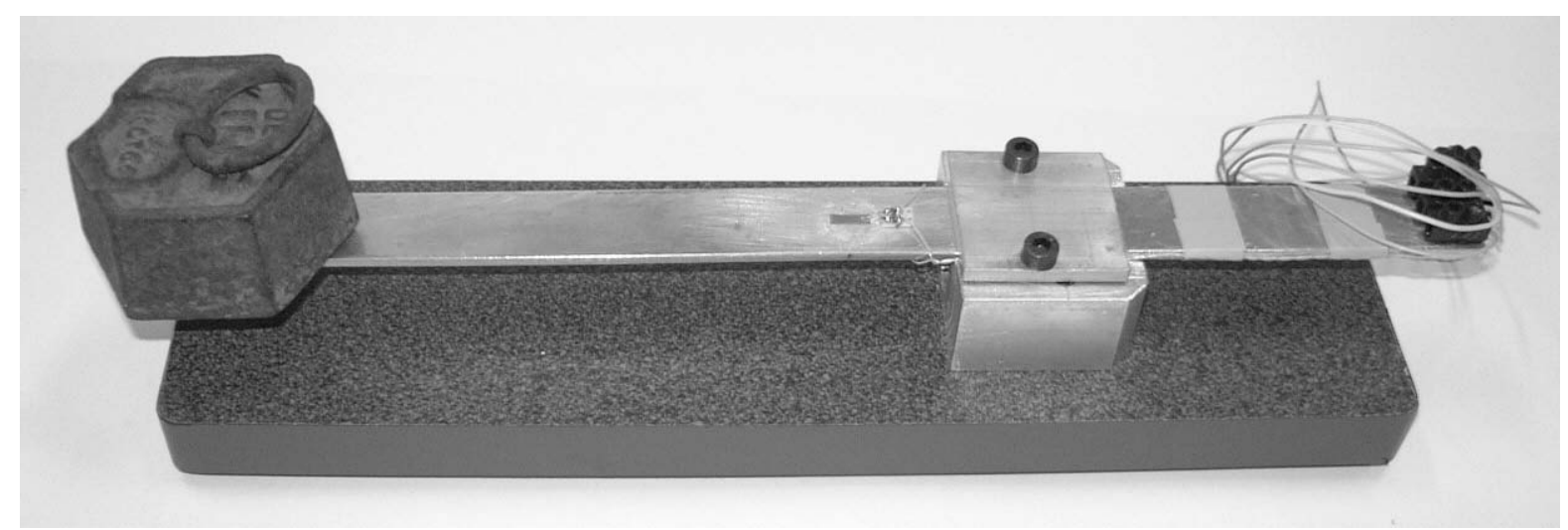

Fig. 1 : Le montage des jauges de contrainte sur une poutre encastrée
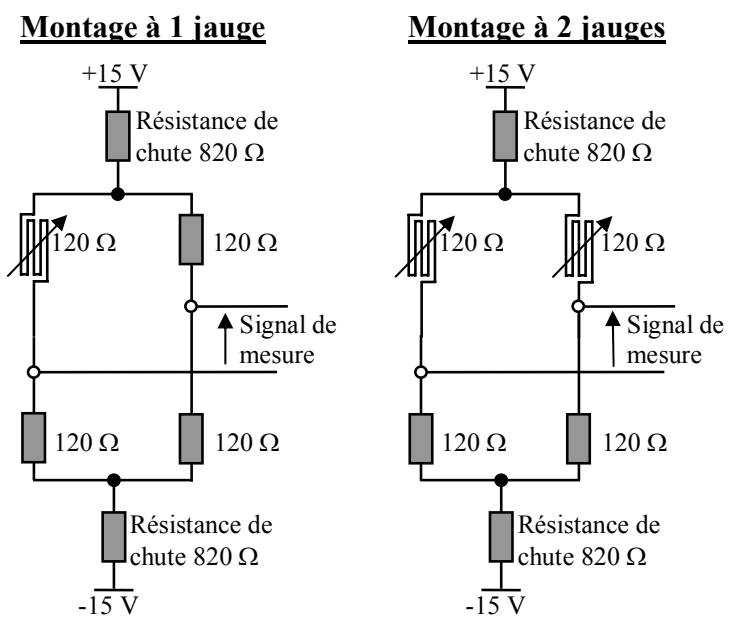

En appliquant une contrainte à la poutre, la variation de la résistance est à peine décelable avec un pont de Wheatstone.

\section{L'ÉLECTRONIQUE}

Pour obtenir une tension de sortie de $10 \mathrm{~V}$ pour une masse de $1 \mathrm{~kg}$, il est nécessaire d'avoir une amplification globale du signal de sortie du pont de l'ordre de 10000 . Si nous répartissons cette amplification sur trois étages nous obtenons une amplification de l'ordre de 22 par étage facilement réalisable par une électronique à amplificateurs opérationnels. Le schéma fonctionnel est présenté figure 3 et ne comporte pas de difficultés particulières de compréhension.

$\underline{\text { Montage à } 4 \text { jauges }}$
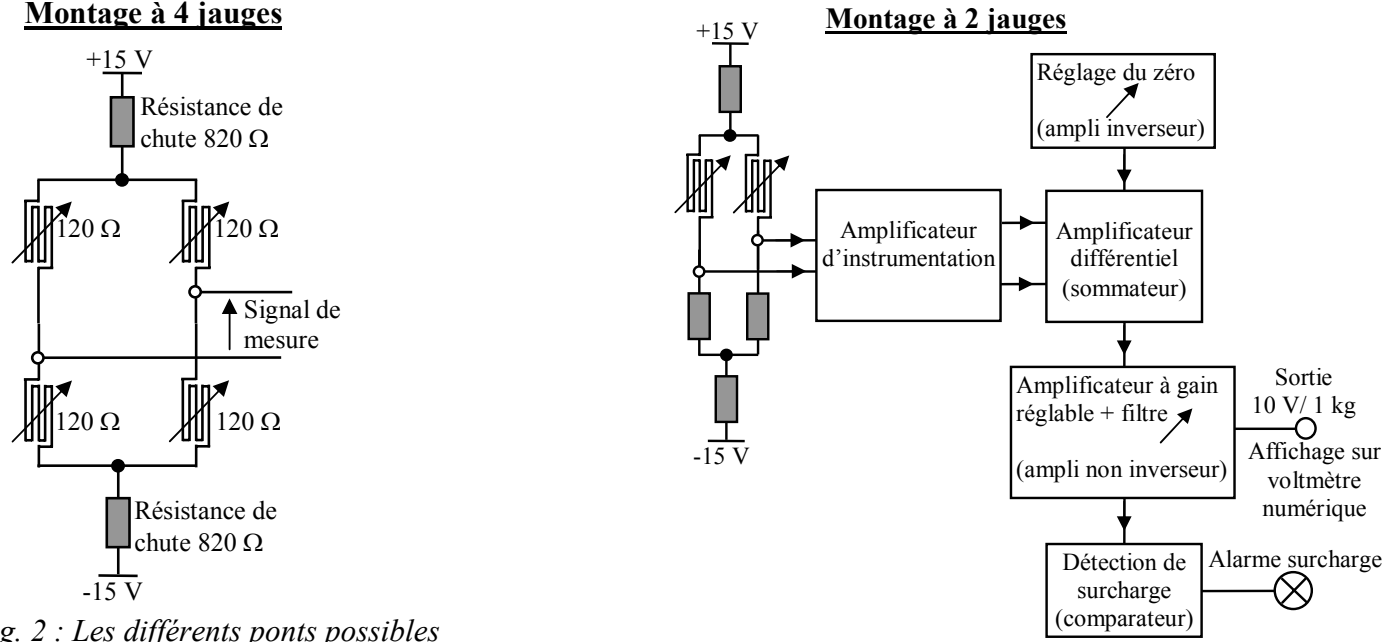

Fig. 3 : Synoptique de l'électronique associée aux jauges

Malheureusement le nombre limité de séances ne nous permet pas d'initier nos étudiants de génie électrique aux notions de résistance des matériaux.

Cependant, nous présentons aux étudiants le phénomène physique de variation de la résistance lié à l'allongement ou au raccourcissement des pistes des jauges.

Nous en profitons pour rappeler qu'en fait une jauge de contrainte ne mesure pas une contrainte mais seulement un allongement relatif du matériau sur laquelle elle est collée.

Finalement la fonction principale du circuit en électronique linéaire revient à réaliser une fonction affine par rapport au signal de sortie du pont de Wheatstone $\left(y=a^{*} x+b\right)$ comme illustré figure 4 . Un premier réglage permet d'ajuster le zéro, la balance étant à vide, un deuxième réglage permet d'ajuster la pente, la balance étant chargée par une masse étalon.

Au-delà de $10 \mathrm{~V}$ l'électronique peut saturer ce qui risque d'entraîner une indication fausse au niveau de l'afficheur. De manière à pallier ce 
dysfonctionnement un indicateur de surcharge est ajouté au montage.

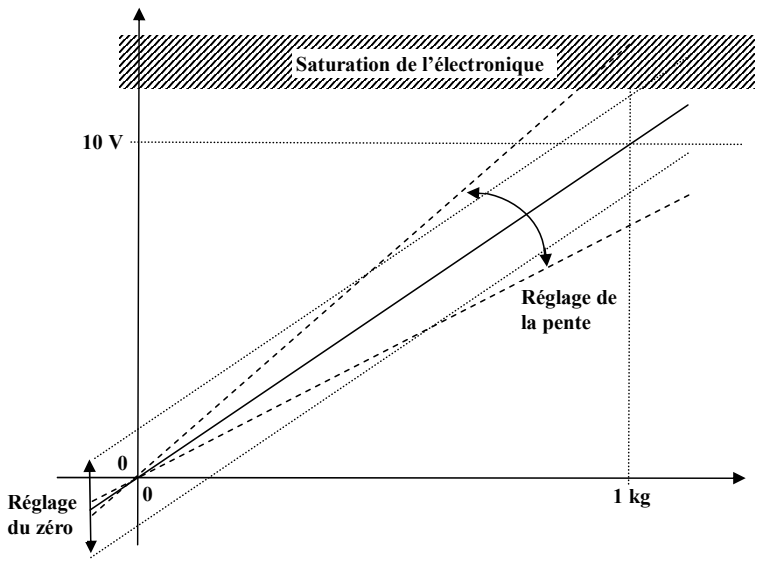

Figure 4 : Fonction affine à réaliser de matière d'électronique

Nous allons maintenant étudier séparément les différentes fonctions composant ce montage électronique.

\subsection{L'amplificateur d'instrumentation}

Pour ne pas perturber le pont de Wheatstone nous utilisons un amplificateur d'instrumentation (fig. 5).

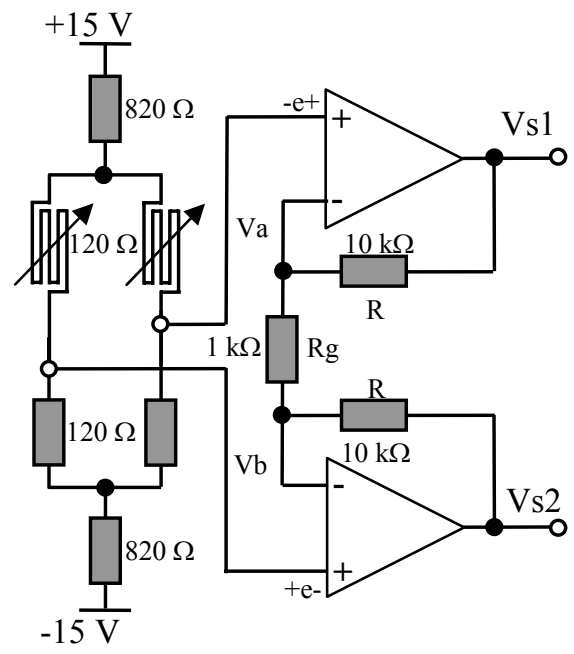

Fig. 5 : L'amplificateur d'instrumentation

Nous avons comme fonction de transfert : Vs1-Vs2 $=\mathrm{G} 1(\mathrm{Va}-\mathrm{Vb}+2 \mathrm{e})$ où e représente la tension de décalage des AOP et $\mathrm{G} 1=[(2 \mathrm{R}+\mathrm{Rg}) / \mathrm{Rg}]$

\subsection{L'amplificateur différentiel}

Pour compenser le décalage et amplifier la différence (Vs1-Vs2), nous utiliserons un amplificateur différentiel (fig. 6).

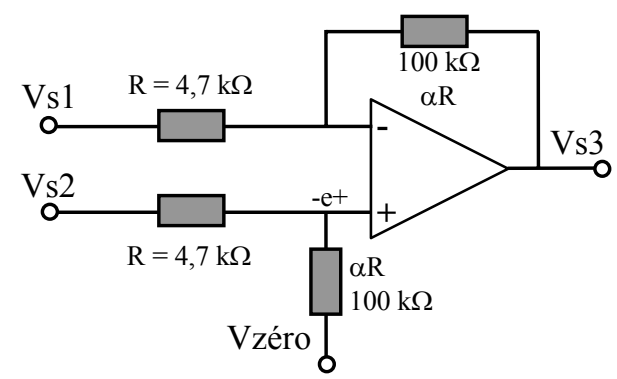

Fig. 6 : L'amplificateur différentiel

Nous obtenons en sortie :

Vs3 $=\alpha(V s 2-V s 1)+(1+\alpha) e+$ Vzéro

\subsection{Le décalage réglable (réglage du zéro)}

Pour compenser la tension de décalage il est nécessaire de générer une tension réglable. Nous utiliserons le montage de la figure 7.

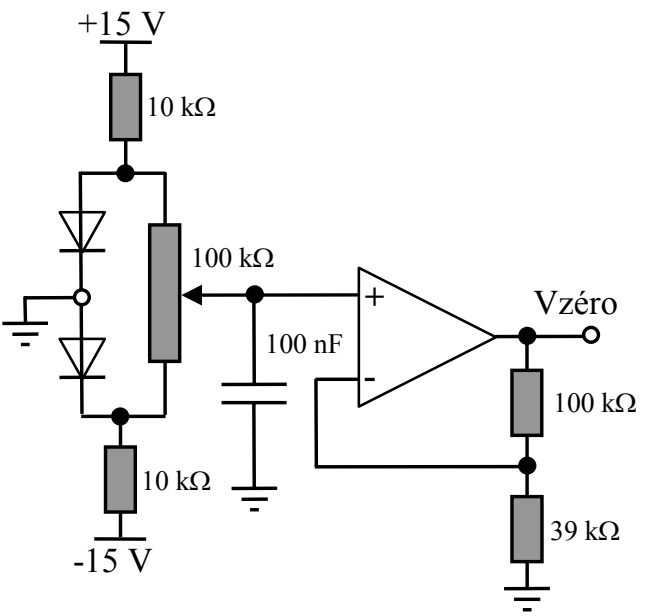

Fig. 7 : Le décalage réglable (Réglage du zéro)

Les erreurs seront compensées pour :

$\mid$ Vzéro $\mid=\alpha \mathrm{G} 1(\Delta \mathrm{Rp} / \mathrm{Rp})+2 \alpha \mathrm{G} 1 \mathrm{e}+(1+\alpha) \mathrm{e} \# 7,5 \mathrm{~V}$. Dans la pratique, les tensions de décalage sont dans le même sens, la compensation nécessaire est bien plus faible.

\subsection{L'amplificateur à gain réglable}

Le dernier étage comporte un amplificateur à gain réglable (fig. 8). Notons que le potentiomètre ne figure pas dans la boucle de contre réaction de l'AOP.

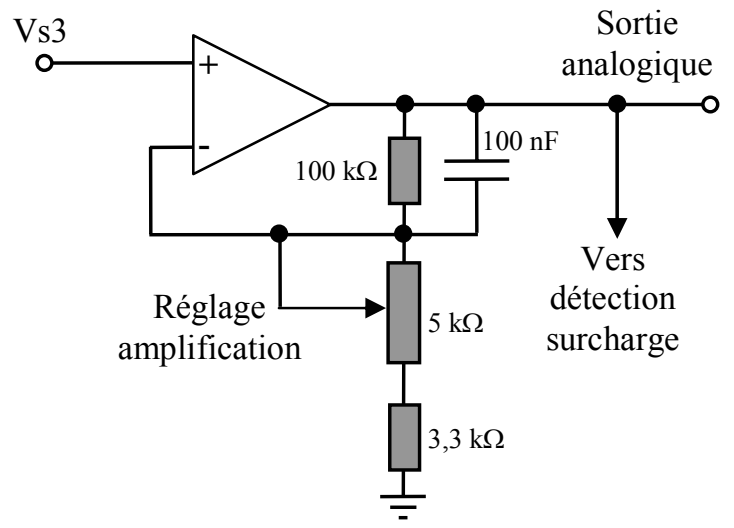

Fig. 8 : L'amplificateur à gain réglable 
Le condensateur de $100 \mathrm{nF}$ associé à la résistance de $100 \mathrm{k} \Omega$ réalise une fonction de filtrage de la tension de sortie.

\subsection{La détection de surcharge}

Afin de détecter une surcharge du montage pouvant induire un affichage erroné, nous utilisons un AOP en comparateur. L'affichage de la surcharge est effectué à l'aide d'une LED rouge, la seconde diode assure la protection en tension inverse de la LED. Cette seconde diode peut être remplacée par une LED verte, le couple de LED indiquant la validité ou la non validité des mesures effectuées.

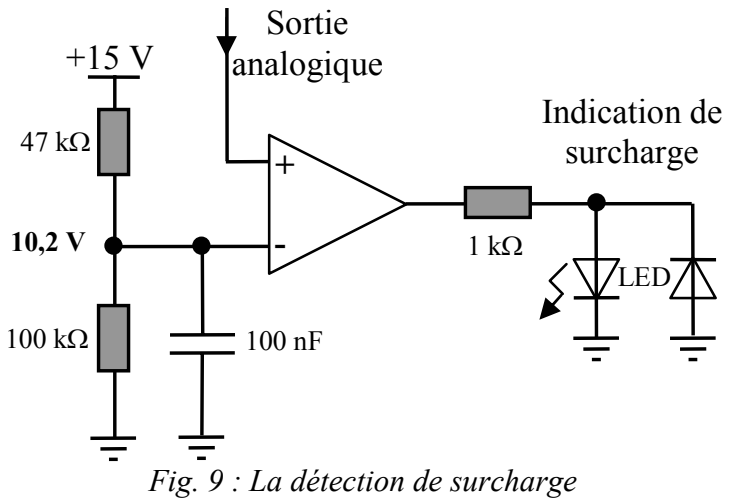

Fig. 9 : La détection de surcharge

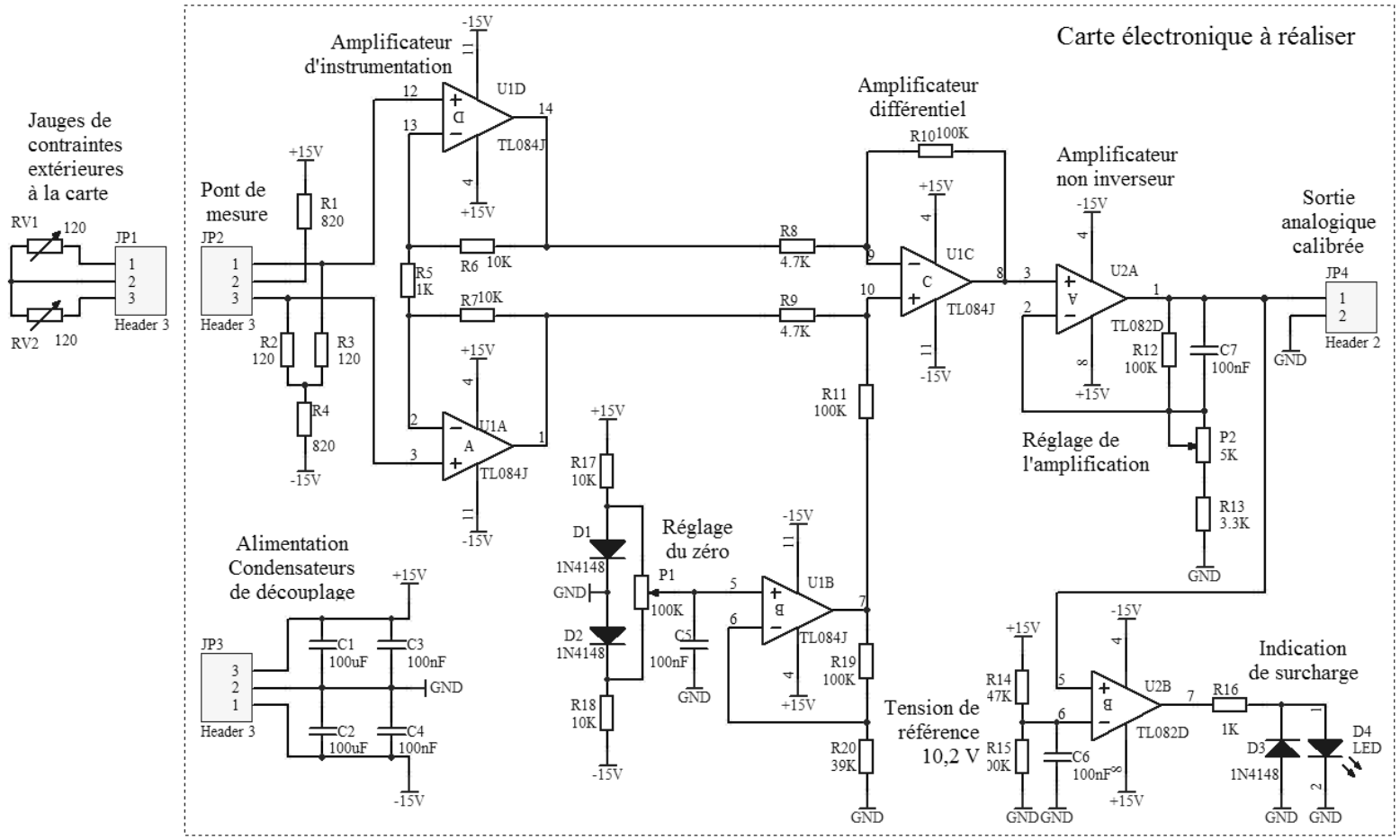

Fig. 10 : Le schéma global du montage

\section{CONCLUSION}

Ce projet très concret permet d'effectuer une synthèse des différents montages à amplificateurs opérationnels : amplificateur d'instrumentation, amplificateur différentiel, amplificateur non inverseur, comparateur.

Une part de ce projet est consacrée à la prise en main d'un logiciel de C.A.O. par les étudiants, en partant de la saisie de schéma, en passant par la simulation analogique et allant jusqu'au routage du circuit imprimé.

Ce projet permet la mise en œuvre d'un ensemble de composants électroniques : résistances potentiomètres, condensateurs, diodes, LED, connecteurs.

Ce projet est également l'occasion d'aborder des problèmes technologiques comme les défauts et les tolérances des différents composants, les réglages par potentiomètres, le découplage des alimentations, l'implantation et l'accessibilité des différents éléments, etc.

La sensibilité du montage réalisé permet de mesurer la masse d'un morceau de sucre (1 gramme). De plus, si nous retournons le montage nous pouvons voir l'influence de la gravité terrestre sur la poutre soumise à son propre poids.

La présentation aux étudiants dès la première séance de la maquette à réaliser et la démonstration de son fonctionnement (grande sensibilité et influence de la gravité terrestre), suscitent des motivations dès le début du projet. 